\title{
EDITORIAL
}

\section{Diskussion belebt das Geschäft}

\author{
Traugott Scheytt · Gudrun Massmann
}

Eingang des Beitrages: 11.3.2015 / Online veröffentlicht: 24.4.2015

(C) Springer-Verlag Berlin Heidelberg 2015

Was sind die großen Themen rund um das Grundwasser in dieser Zeit? Auf diese Frage mag jeder eine etwas andere Antwort geben, abhängig von der Art der Beschäftigung mit dem Grundwasser, abhängig von der betrachteten Region und abhängig von dem eigenen fachlichen Hintergrund.

Auf jeden Fall haben viele das Grundwasser für sich entdeckt, darunter auch viele, die keine vertieften hydrogeologischen Fachkenntnisse haben. Und natürlich lassen sich viele Fragestellungen auch bearbeiten, wenn man die lokalen Strömungsverhältnisse nicht kennt oder von der hydrochemischen Zonierung keine Vorstellung hat. Zudem erscheint es, als ob die großen gesellschaftlich relevanten hydrogeologischen Fragestellungen eher von außen herangetragen werden, als aus der eigenen Arbeit zu erwachsen. $\mathrm{Zu}$ diesen Themen gehören zum Beispiel $\mathrm{CO}_{2}$-Abscheidung und -Speicherung (eher bekannt unter CCS), das Hydraulic Fracturing (,Fracking“) oder Themen rund um die Tiefe Geothermie und die wieder aufkommende Diskussion zur Endlagerung von radioaktiven Abfällen. Die Anzahl an Fachbeiträgen in der Zeitschrift Grundwasser zu diesen Themen ist mehr als überschaubar, obwohl hydrogeologische Fragestellungen ganz direkt betroffen sind. Dies war auch bereits in der Vergangenheit nicht viel anders: Als Beispiel sei die große BMBF-Fördermaßnahme KORA (Kontrollierter Rückhalt und Abbau) mit vielen beteiligten Ingenieurbüros und Universitäten genannt, die nur eine marginale Anzahl an Veröffentlichungen in Grundwasser erbrachte. Wir sind jedoch davon überzeugt, dass die Leserinnen und Leser sehr gerne erfahren würden, welche unterschiedlichen Methoden angewandt werden, wie die Ergebnisse aussehen und welche Lehren zu ziehen sind.

Daher haben wir nun zu einem Grundwasser-Sonderheft aufgerufen, das speziell Themen aufgreift, die in der Gesellschaft kontrovers diskutiert werden: CCS, Fracking und Tiefe Geothermie. Das Team von Grundwasser hofft, dass das Heft zu einer lebendigen fachlichen Diskussion führen wird. Es soll bei den Beiträgen zu diesem Sonderheft weniger um Dokumentation, als um Kommunikation gehen. Die oben genannten Themen sind auch nur als Beispiele für eine ganze Reihe denkbarer Themen zu sehen. Gut geschriebene Veröffentlichungen zu aktuellen und relevanten Themen sind für alle Leserinnen und Leser interessant, schärfen den Blick für die kritischen Fragen, wecken die Aufmerksamkeit und lassen die Zeitschrift Grundwasser zu einer Pflichtlektüre für alle hydrogeologisch interessierten Fachleute werden. Wir hoffen, dass es nicht bei diesem einen Sonderheft bleibt, sondern in Zukunft weitere Sonderhefte folgen und die Diskussionen um das Grundwasser beleben werden!

Ihre

Gudrun Massmann und Traugott Scheytt

PD Dr. T. Scheytt $(\bowtie)$

TU Berlin,

Ernst-Reuter-Platz 1, 10587 Berlin, Deutschland

E-Mail: traugott.scheytt@tu-berlin.de

Prof. Dr. G. Massmann

Carl von Osietzky Universität,

26111 Oldenburg, Deutschland

E-Mail: gudrun.massmann@uni-oldenburg.de 